\section{Dengue complicado y miocarditis: comunicación de un caso}

María Gracia Pereda, Marianelly López y Melissa Mariluz

\section{Myocarditis and complicated dengue: a case report}

Hemorragic dengue fever is a prevalent infection in many countries around the world. Myocarditis is a severe manifestation of dengue virus infection. With prompt intervention and an early diagnosis, the outcome of this condition can be improve. We report a adult patient with complicated dengue, myocarditis, cardiac and respiratory insufficiency with acute renal injury.

Key words: Dengue, myocarditis.

Palabras clave: Dengue, miocarditis.

\section{Introducción}

El dengue es la virosis transmitida por vector más prevalente en la actualidad; estimándose más de 50 millones de infecciones por año ${ }^{1}$. Esta enfermedad, puede cursar con sintomatología leve con presencia de fiebre moderada, cefalea, dolor retro-ocular, dolor muscular, y, en menos de $50 \%$ de los casos, exantema pruriginoso, hasta sintomatología grave con disfunción orgánica ${ }^{2}$.

\section{Caso clínico}

Comunicamos el caso de un paciente varón de 22 años de edad, procedente de Iquitos, selva peruana, sin antecedentes de importancia. Tenía una historia de cuatro días de evolución de sensación febril, calofríos, cefalea, epigastralgia y gingivorragia. Al examen físico presentaba una temperatura de $37,5^{\circ} \mathrm{C}$, presión arterial de $110 / 80 \mathrm{~mm} \mathrm{Hg}$ y frecuencia respiratoria de 22 respiraciones por min. El resto del examen no tenía alteraciones. Al día siguiente, se agregó distensión abdominal, disnea a mediano esfuerzo y deposiciones con contenido rojizo oscuro, cuyo cultivo fue negativo; al examen físico pulmonar presentaba un murmullo vesicular disminuido en el tercio inferior, sin ruidos agregados. Dentro de los parámetros de laboratorio se encontró un hematocrito de $48 \%$ y plaquetas en $51.000 / \mathrm{mm}^{3}$. Se le realizó serología para dengue y leptospira, resultando la IgM para dengue positiva. Se confirmó el diagnóstico de dengue con una segunda muestra de IgM. Por la aparición de disnea se realizó una ecocardiografía (Figura 1) y troponina que resultó positiva y que configuró el diagnóstico de una miocarditis. En la radiografía de tórax se encontró una cardiomegalia con derrame pleural

Universidad Peruana de Ciencias Aplicadas.

No existen conflictos de interés

No presento fuente de financiamiento.

Recibido: 27 de noviembre de 2014 / Aceptado: 3 de marzo de 2015

Correspondencia a:

María Gracia Perada

mariagraciapereda@gmail.com
(Figura 2). Se ingresó a Unidad de Cuidados Intensivos por mala evolución y persistencia de la fiebre. Por insuficiencia respiratoria y compromiso de conciencia (Glasgow 10) se conectó a ventilación mecánica invasora. Dentro de los exámenes auxiliares destacaban gases arteriales: $\mathrm{pH} 7,52 ; \mathrm{pCO}_{2}$ $58 \mathrm{mmHg}$ y $\mathrm{PO}_{2}$ (A-a) $368 \mathrm{mmHg}$, recuento de plaquetas $157.000 / \mathrm{mm}^{3}$, hematocrito $43 \%$, glucosa $167 \mathrm{mg} / \mathrm{dl}$ y creatinina $1,08 \mathrm{mg} / \mathrm{dl}$. Se diagnosticó dengue grave con injuria renal aguda, insuficiencia cardíaca, respiratoria y sepsis grave. Se inició tratamiento con ciprofloxacina, ceftazidima en forma empírica, vitamina $\mathrm{K}$ y furosemida i.v. A los dos días, el paciente evolucionó hacia la mejoría, con ventilación espontánea y disminución del edema. Dentro de los exámenes el control de troponina fue negativo y creatinina dentro de rangos normales. Se realizó una ecocardiografia de control a la semana de evolución que resultó normal.

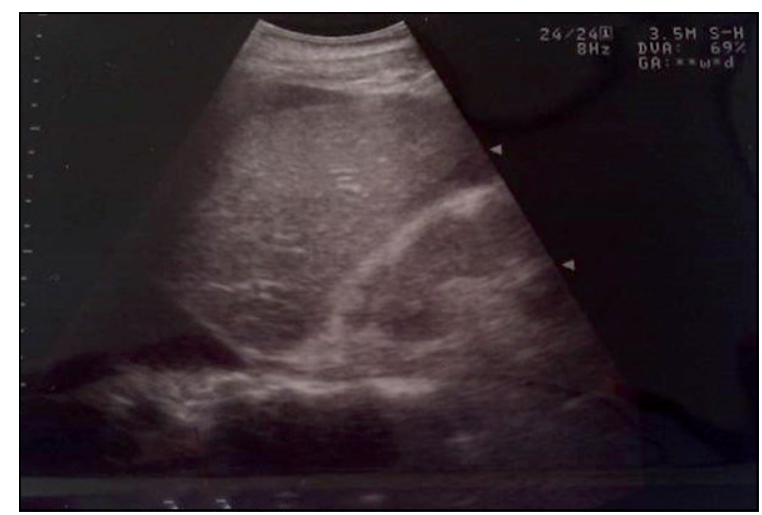

Figura 1. Paciente de 22 años con diagnóstico de miocarditis. Se aprecia engrosamiento de las paredes ventriculares.

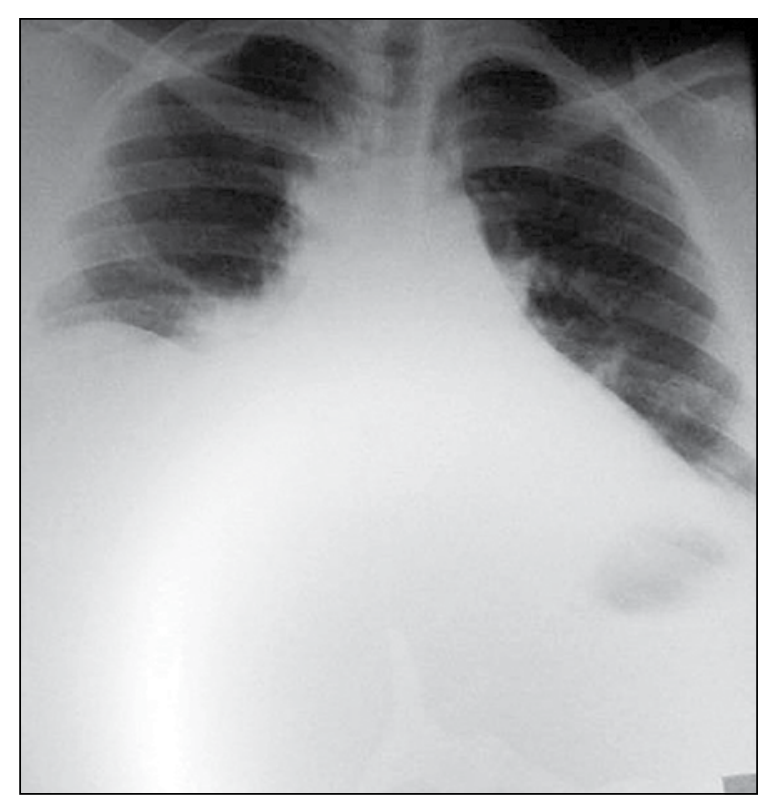

Figura 2. Radiografía de tórax $A P$, se aprecia derrame pleural y presencia de cardiomegalia. 


\section{Discusión}

La clasificación de dengue complicado es dificultosa debido a que existen pacientes que no cumplen con todos los criterios diagnósticos, especialmente si presentan complicaciones atípicas de la enfermedad. La ausencia de fiebre dentro de las manifestaciones clínicas, aunque ésta se encuentre en $90 \%$ de los casos, no deberían excluir dicho diagnóstico ${ }^{4}$. Dentro de las presentaciones atípicas y poco frecuentes, las cuales en su mayoría son clasificadas como dengue grave, destacan la encefalitis, miocarditis, hepatopatía, y la insuficiencia renal aguda ${ }^{5}$. El daño miocárdico es una entidad muy infrecuente, y es consecuencia directa de la invasión viral en las fibras musculares ${ }^{3}$. La sintomatología es inespecífica y la elevación de los biomarcadores cardíacos no se encuentran presentes en todos los pacientes, motivo por el cual, si es que existe desconocimiento de dicha asociación, puede llevar a un mal enfoque. El diagnóstico de miocarditis se realiza al detectar una disfunción ventricular por medio de una ecocardiografía, incluso cuando esta es subclínica. Evidencia clínica y ecocardiográfica de disfunción miocárdica es suficiente para ingresar al paciente a una Unidad de Cuidados Intensivos ya que existe evidencia de mayor riesgo de shock cardiogénico y muerte ${ }^{3}$.

El equipo de salud debe realizar un seguimiento estricto del paciente y observar signos de alarma por lo menos $48 \mathrm{~h}$ de finalizada la etapa febril ${ }^{5}$. Existen signos predictores de dengue, que ayudan al diagnóstico del mismo, como la presencia de leucopenia, distensión abdominal, duración de la fiebre, entre otros ${ }^{6}$. Es importante que todo centro de salud esté en conocimiento de esto para hacer un diagnóstico y tratamiento temprano. Los pacientes que llegan a esta etapa sin un diagnóstico y tratamiento, tienen una mor- talidad de 30 a $50 \%{ }^{5}$, por tal motivo se recomienda descartar potenciales complicaciones del dengue a todo paciente con este diagnóstico mediante una ecografía abdominal y ecocardiografia. Asimismo, existe evidencia que pacientes con dengue grave, después de su curación, pueden evolucionar con deterioro cardíaco, de predominio ventricular, por lo que es importante hacer un adecuado seguimiento ${ }^{3}$. En este caso es indispensable realizar un examen físico detallado y así detectar hallazgos importantes que pueden pasar desapercibidos y hacer énfasis en la valoración médica integral.

\section{Referencias bibliográficas}

1.- Kroeger A, Nathan M, Hombach J, World Health Organization TDR Reference Group on Dengue. Dengue. Nat Rev Microbiol 2004; 2: 360-1.

2.- Teixeira M G, Barreto M L. Diagnosis and management of dengue. BMJ 2009; 339: b4338.

3.- Yacoub S, Griffiths A, Chau T T, Simmons C P, Wills B, Hien T T, et al. Cardiac function in Vietnamese patients with different dengue severity grades. Crit Care Med 2012; 40: 477-83.

4.- Gregory C J, Santiago L M, Argüello D F, Hunsperger E, Tomashek K M. Clinical and laboratory features that differentiate dengue from other febrile illnesses in an endemic area-Puerto Rico, 2007-2008. Am J Trop Med Hyg 2010; 82: 922-9.

5.- Jaenisch T, Wills B. Resultado del estudio DENCO. TDR/WHO Reunión de Expertos en la Clasificación y Manejo de casos de Dengue. Implicaciones del Estudio DENCO. WHO, Ginebra, Sep 30-Oct. 1/2008.

6.- Carrasco L R, Leo Y S, Cook A R, Lee V J, Thein T L, Go C J. Predictive tools for severe dengue conforming to World Health Organization 2009 Criteria. PLoS Negl Trop Dis 2014; 8: e2972. 